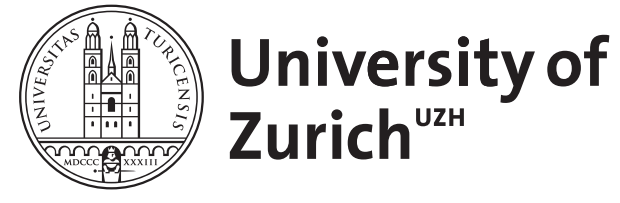
Archive

University of Zurich

University Library

Strickhofstrasse 39

CH-8057 Zurich

www.zora.uzh.ch

Year: 2013

\title{
Fatal Neisseria sicca endocarditis
}

Sommerstein, R ; Ramsay, D ; Dubuis, O ; Waser, S ; Aebersold, F ; Vogt, M

DOI: https://doi.org/10.1007/s15010-012-0393-8

Posted at the Zurich Open Repository and Archive, University of Zurich

ZORA URL: https://doi.org/10.5167/uzh-70775

Journal Article

Published Version

Originally published at:

Sommerstein, R; Ramsay, D; Dubuis, O; Waser, S; Aebersold, F; Vogt, M (2013). Fatal Neisseria sicca endocarditis. Infection, 41(3):747-749.

DOI: https://doi.org/10.1007/s15010-012-0393-8 


\title{
Fatal Neisseria sicca endocarditis
}

\author{
R. Sommerstein • D. Ramsay $\cdot$ O. Dubuis • \\ S. Waser $\cdot$ F. Aebersold $\cdot$ M. Vogt
}

Received: 26 October 2012/ Accepted: 16 December 2012/Published online: 8 January 2013

(C) Springer-Verlag Berlin Heidelberg 2013

\section{Dear Editor}

A 75-year-old previously healthy woman was admitted to our hospital with a subacute stroke. Before, she had complained of unspecific symptoms including arthralgia, myalgia, cephalgia and vertigo of 4-week duration. Despite absence of an obvious infectious focus, an empiric outpatient treatment at a different clinic with ciprofloxacin and then doxycycline was administered but shown to be ineffective. At admission, the patient was in a diminished general state but afebrile. There were signs of right sided hemiparesis and a slurred speech. She was hypotensive and tachycardic but there were no signs of congestive heart failure. Clinically, no infectious focus was detectable. A CT scan of the brain (Fig. 1a) confirmed several hypointense cortical lesions. Kidney sonography (Fig. 1b) to investigate microhematuria showed a circumscript lesion on the lower left pole (arrow) most likely corresponding to a kidney infarct. Trans-esophagal echocardiography (TEE) (video) revealed large polypoid vegetations on the aortic valve. Neisseria sicca grew in six of six blood cultures and was confirmed upon sequencing of the $16 \mathrm{~S}$ rRNA gene.

Electronic supplementary material The online version of this article (doi:10.1007/s15010-012-0393-8) contains supplementary material, which is available to authorized users.

R. Sommerstein $(\bowtie) \cdot$ D. Ramsay $\cdot$ S. Waser $\cdot$ M. Vogt Department of Internal Medicine, Cantonal Hospital Zug,

Zug, Switzerland

e-mail: rami.sommerstein@usz.ch

O. Dubuis

Viollier AG, Basel, Switzerland

\section{F. Aebersold}

Department of Pathology, Cantonal Hospital Lucerne, Lucerne, Switzerland
The patient deteriorated rapidly despite adequate antibiotic therapy with amoxicillin/clavulanic acid and gentamicin with consecutive embolic phenomena occurring in the skin (Fig. 1c), the cerebellum (Fig. 1d), the conjunctives (Fig. 1e) and the lungs (Fig. 1f). Surgery was declined by the patient and she eventually died 8 days after admission. Post mortem examination confirmed endocarditis of the aortic valve (Fig. 1g), kidney infarction (Fig. 1h) and embolism into further sites.

Infective endocarditis is a life threatening disease and the mortality currently remains high at around $15 \%$ [1]. To our knowledge only 17 cases of endocarditis caused by Neisseria sicca have been described in the literature [2]. The Gram-negative diplococcus Neisseria sicca is a member of the family Neisseriaceae and a commensal inhabitant of the human upper respiratory tract [3].

In this patient, there was no clinical or radiological evidence of a preceding or concomitant upper respiratory tract infection. These findings were confirmed by post mortem studies and thus provide evidence that Neisseria sicca endocarditis was not caused by spreading of a preexisting upper respiratory tract infection.

Most cases of Neisseria sicca endocarditis have been described in intravenous drug users and patients with underlying heart disease. Prolonged fever and embolic phenomena occurred in the majority of the cases $(>90 \%)$. Surgery was required in half of the patients with Neisseria species endocarditis due to large vegetations with concurrent embolism and destruction of the valve.

In patients rapidly treated with surgical and appropriate antibiotics mortality is reported to be low with good neurologic outcome.

In this case the microbe was susceptible to the empiric amoxicillin/clavulanic acid therapy at a mean inhibitory concentration of $1.0 \mathrm{mg} / \mathrm{l}$ and to ceftriaxone at $0.047 \mathrm{mg} / \mathrm{l}$. 
Fig. 1 Spectrum of the clinical, sonographical, radiological and pathological extent of the disease
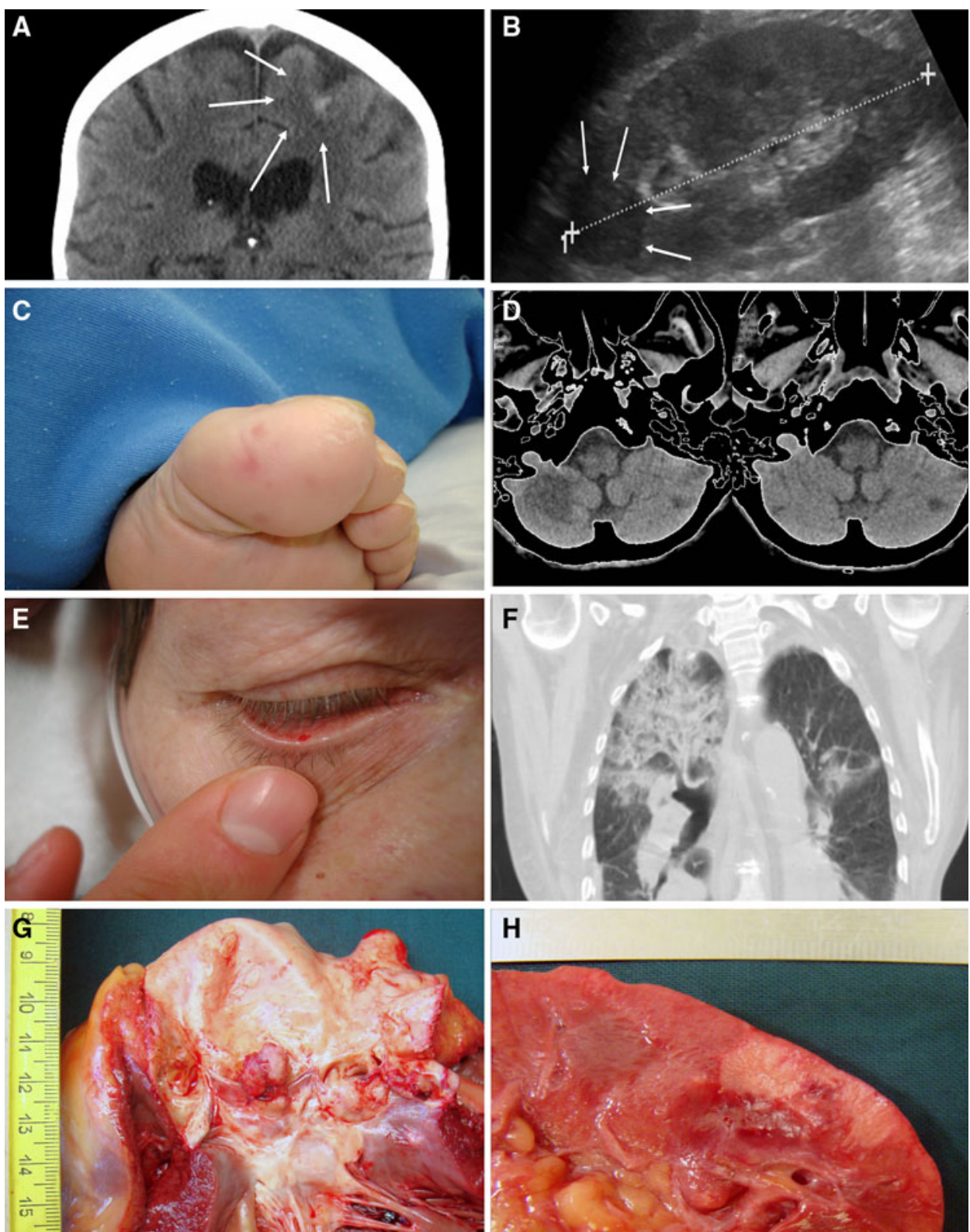

H

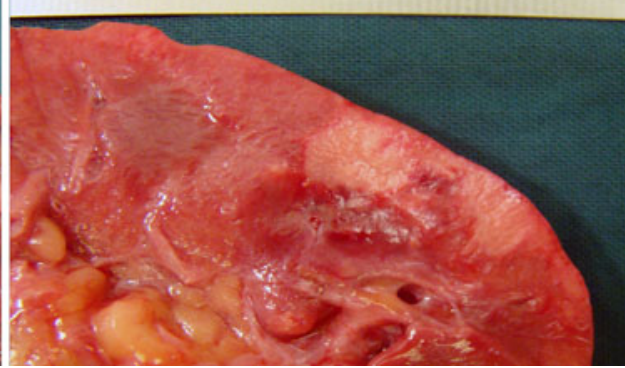

The microbe was intermediary susceptible to ciprofloxacine and resistant to penicllin and rifampicin.

Neisseria sicca has also rarely been described as causative agent in patients with meningitis, discitis, osteomyelitis, septic arthritis and both immunocomprimised and immunocompetent hosts [2, 4-6]. In conclusion we describe for the first time for this rare entity a post mortem analysis that enables to link clinical, radiological and microbiological data to classical autopsy findings.

\section{Informed consent}

The patient gave informed consent that

- the information will be published without name attached and Infection will make every attempt to ensure anonymity. However, complete anonymity cannot be guaranteed. It is possible that somebody somewhere may identify the patient.

- The information may be published in the print Infection which is distributed worldwide. The journal goes mainly to doctors but is seen by many non-doctors, including journalists.

- The information may also be placed on the Infection website.

- Infection will not allow the information to be used for advertising or packaging or to be used out of context (for example, a photograph will not be used to illustrate an article that is unrelated to the subject of the photograph.)

Acknowledgments We would like to thank Prof. Rainer Weber, MD from the Clinic of Infectious Diseases and Hospital Hygiene, 
Department Internal Medicine, University Hospital of Zurich, Switzerland for critical proofreading of the Manuscript. This work was supported by the Hans-Paul Wälchli Foundation for clinical research (Lugano, Switzerland). The funding source had no role in any stage of the manuscript development.

Conflict of interest The authors declare that there is no conflict of interest that interferes with the publication of this report. There are no financial conflicts of interest in this report.

\section{References}

1. Leone S, Ravasio V, Durante-Mangoni E, Crapis M, Carosi G, Scotton PG, et al. Epidemiology, characteristics, and outcome of infective endocarditis in Italy: the Italian study on endocarditis. Infection. 2012;40:527-35. doi:10.1007/s15010-012-0285-y.

2. Aronson PL, Nelson KA, Mercer-Rosa L, Donoghue A. Neisseria sicca endocarditis requiring mitral valve replacement in a previously healthy adolescent. Pediatr Emerg Care. 2011;27:959-62. doi: 10.1097/PEC.0b013e3182309e9500006565-201110000-00016.

3. Shaw FW. The pathogenicity of Neisseria sicca. Science. 1932;75:488. doi:75/1949/48810.1126/science.75.1949.488.

4. Jeurissen A, Stroy JP, Wielenga RP, Andriesse GI. Severe infective endocarditis due to Neisseria sicca: case report and review of literature. Acta Clin Belg. 2006;61:256-8.

5. Heiddal S, Sverrisson JT, Yngvason FE, Cariglia N, Kristinsson KG. Native-valve endocarditis due to Neisseria sicca: case report and review. Clin Infect Dis. 1993;16:667-70.

6. Gay RM, Sevier RE. Neisseria sicca endocarditis: report of a case and review of the literature. J Clin Microbiol. 1978;8:729-32. 